\title{
A Promising New Device for the Prevention of Parastomal Hernia
}

Surgical Innovation

2015, Vol. 22(3) 283-284

(C) The Author(s) 2014

Reprints and permissions:

sagepub.com/journalsPermissions.nav DOI: 10.1 | $77 / \mid$ 5533506/4560270

sri.sagepub.com

\author{
Henry Hoffmann, MD', Daniel Oertli, MD, FACS', Savas Soysal, MD', \\ Urs Zingg, $\mathbf{M D}^{2}$, Dieter Hahnloser, $\mathbf{M D}^{3}$, and Philipp Kirchhoff, MD'
}

\begin{abstract}
Parastomal hernia (PSH) is the most frequent long-term stoma complication with serious negative effects on quality of life. Surgical revision is often required and has a substantial morbidity and recurrence rate. The development of PSH requires revisional surgery with a substantial perioperative morbidity and high failure rate in the long-term followup. Prophylactic parastomal mesh insertion during stoma creation has the potential to reduce the rate of PSH, but carries the risk of early and late mesh-related complications such as infection, fibrosis, mesh shrinkage, and/or bowel erosion. We developed a new stomaplasty ring (KORING), which is easy to implant, avoids potential mesh-related complications, and has a high potential of long-term prevention of PSH. Here we describe the technique and the first use.
\end{abstract}

\section{Keywords}

biomedical engineering, colorectal surgery, hernias, surgical oncology

\section{Introduction}

Parastomal hernia (PSH) is the most frequent long-term complication with serious negative effects on quality of life ${ }^{1}$ following stoma formation. PSH appears most commonly within the first 2 years after stoma formation with a prevalence of $35 \%$ to $50 \% .{ }^{2} \mathrm{PSH}$ often requires surgical repair with high morbidity and recurrency rates. ${ }^{3}$ Preventing PSH by prophylactic mesh insertion during stoma creation remains a challenging procedure, including tissue dissection, carrying the risk of serious meshrelated complications. ${ }^{4,5}$ Therefore, we developed a new stomaplasty ring (KORING), which is easy to implant, requiring limited tissue dissection and avoids potential mesh-related complications providing a high potential of long-term prevention of PSH.

\section{Description of the Device}

The new stomaplasty ring (KORING) consists of a nonabsorbable and immunologically inert material (polyethylene terephthalate) with a flexible but not dilatable ring (Figure 1). The device is available in different diameters $(2.5 \mathrm{~cm}, 3.5 \mathrm{~cm}$, and $4.5 \mathrm{~cm})$ enabling precise fitting to the stoma site. The KORING is sutured to the anterior rectus fascia, which distributes axial forces evenly to the ring preventing weakness of the fascia (Figure 1). The lip of the ring is inserted between the rectus muscle and the peritoneum to prevent direct contact of the ring with other bowel loops, avoiding bowel entrapment and adhesions (Figure 2).

\section{The Operation}

At the designated stoma site a circular skin incision is performed followed by blunt dissection through the subcutaneous tissue. Then a second circular incision is made in the external rectus fascia, the rectus muscle is preserved by blunt separation. Subsequently, the posterior rectus sheath (applicable cranial to the linea arcuata) and the peritoneum are incised. After choosing the correct diameter the ring is fixed to the external rectus fascia with 8 to 10 interrupted, nonabsorbable sutures to prevent constriction of the ring (purse-string effect).

The lip seal of the ring is then fitted between the peritoneum and the muscle (Figure 2). The intestine is then pulled through the ring and fixed according to common recommended practices to form the external orifice.

\footnotetext{
'University Hospital Basel, Switzerland

${ }^{2}$ Limmattal Hospital, Schlieren, Switzerland

${ }^{3}$ University Hospital Lausanne, Switzerland

Corresponding Author:

Henry Hoffmann, University Hospital Basel, Spitalstrasse 2I, 403I

Basel, Switzerland.

Email: henry.hoffmann@usb.ch
} 


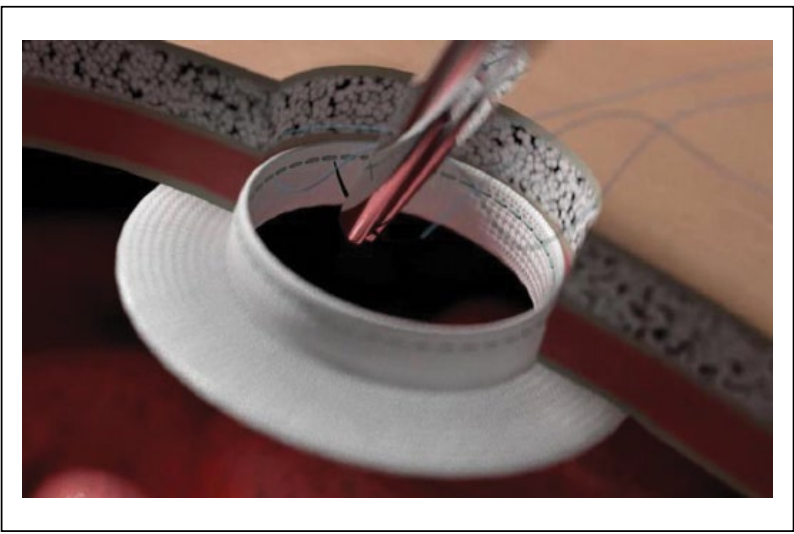

Figure I. The stomaplasty ring (KORING) and its placement in the abdominal wall.

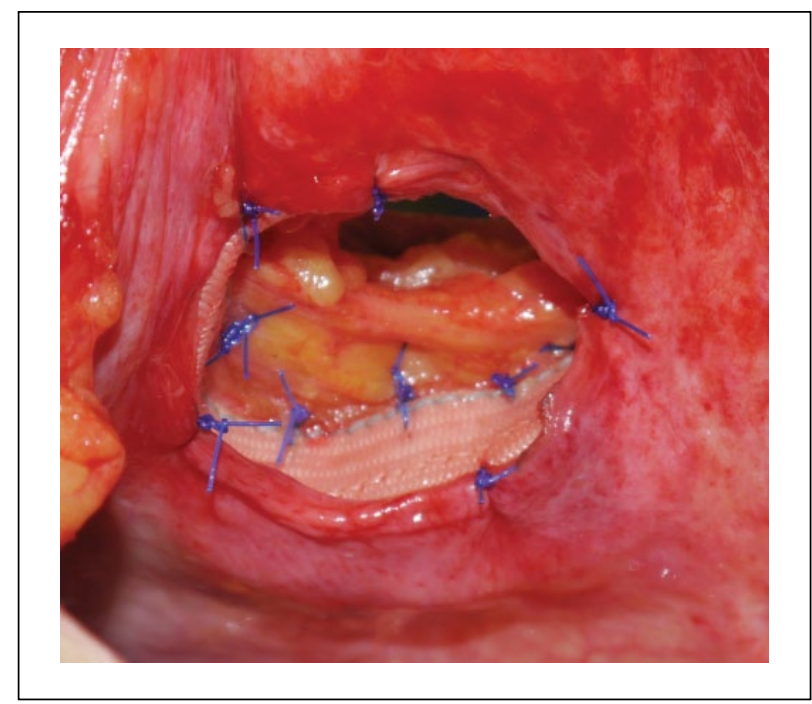

Figure 2. The KORING after implantation (peritoneal perspective).

\section{Outcomes}

The device was first used in a 76-year-old man undergoing abdominoperineal resection of the rectum with formation of an end-colostomy. The implantation of the ring took 10 minutes. No additional tissue mobilization was necessary. The braided structure of the ring made the fixation easy. After implantation the ring had no contact to the other bowel loops in the abdominal cavity and minimal contact with surrounding tissue (Figure 2). The postoperative course was uneventful.

\section{Summary}

Parastomal hernia is a serious stoma-related complication in terms of morbidity and socioeconomic impact. Prophylactic mesh insertion has shown promising results, remains a demanding procedure, and therefore has not yet become standard during stoma formation. The new stomaplasty ring (KORING) offers a simple and intuitive implantation avoiding large tissue dissection. It has no direct contact with the abdominal content and therefore potentially reduces the risk of adhesions and mesh infection. It may have a high impact on prevention of PSH in the future, improving quality of life of stoma patients as well as decreasing health care costs by preventing further surgical interventions due to PSH. Further clinical trials are necessary.

\section{Declaration of Conflicting Interests}

The author(s) declared no potential conflicts of interest with respect to the research, authorship, and/or publication of this article.

\section{Funding}

The author(s) disclosed receipt of the following financial support for the research, authorship, and/or publication of this article: This study was supported by the grant "Clinical Research and Young Academics Advancement" of the University Basel, Switzerland (Ref. No. DMS2214).

\section{References}

1. Kwiatt M, Kawata M. Avoidance and management of stomal complications. Clin Colon Rectal Surg. 2013;26: $112-121$

2. Ripoche J, Basurko C, Fabbro-Perray P, Prudhomme M. Parastomal hernia. A study of the French federation of ostomy patients. J Visc Surg. 2011;148:e435-e441.

3. Helgstrand F, Rosenberg J, Kehlet H, Jorgensen LN, Wara $\mathrm{P}$, Bisgaard T. Risk of morbidity, mortality, and recurrence after parastomal hernia repair: a nationwide study. Dis Colon Rectum. 2013;56:1265-1272.

4. Aziz F, Zaeem M. Chronic abdominal pain secondary to mesh erosion into ceacum following incisional hernia repair: a case report and literature review. J Clin Med Res. 2014;6:153-155.

5. Annaiah TK, Amin T, Webb B. Bowel perforation resulting from mesh erosion: a rare complication following abdominal sacrocolpopexy. J Obstet Gynaecol. 2010;30:744-745. 